\title{
New Constraints on Xenonphobic Dark Matter from DEAP-3600
}

\author{
Carlos E. Yaguna* \\ Escuela de Fúsica, Universidad Pedagógica y Tecnológica de Colombia, \\ Avenida Central del Norte \# 39-115, Tunja, Colombia
}

\begin{abstract}
The first-year results from DEAP-3600, a single-phase liquid argon direct-detection dark matter experiment, were recently reported. At first sight, they seem to provide no new constraints, as the limit lies well within the region already excluded by three different xenon experiments: LUX, PandaX-II, and XENON1T. We point out, however, that this conclusion is not necessarily true, for it is based on the untested assumption that the dark matter particle couples equally to protons and neutrons. For the more general case of isosping-violating dark matter, we find that there are regions in the parameter space where DEAP-3600 actually provides the most stringent limits on the dark matter-proton spin-independent cross section. Such regions correspond to the so-called Xenonphobic dark matter scenario, for which the neutron-to-proton coupling ratio is close to -0.7 . Our results seem to signal the beginning of a new era in which the complementarity among different direct detection targets will play a crucial role in the determination of the fundamental properties of the dark matter particle.
\end{abstract}

\section{Introduction}

Determining the fundamental nature of the dark matter particle is one of the most important open problems in particle and astroparticle physics today $[1,2]$. In recent years, direct detection experiments have been able to probe new regions of parameter space and to set stringent limits on the dark matter interactions [3-6]. Unfortunately, the results from direct detection experiments continue to be presented, probably due to historical reasons, in a way that may hinder their true relevance.

Typically, direct detection constraints are shown in terms of the so-called dark matter-nucleon cross section, which differs from the physically meaningful dark matter-proton cross section. They would coincide if the dark matter

\footnotetext{
*carlos.yaguna@uptc.edu.co
} 
coupled equally to protons and neutrons, but such an assumption is not supported either theoretically or experimentally. In models where the dark matter couples differently to protons and neutrons -the so-called isospin-violating scenario [7-11]- it does not make sense to compare the limits on the dark matternucleon cross section obtained from different targets, as is usually done in the literature. Instead, one should compare the limits on the dark matter-proton cross sections, which will depend on the neutron-to-proton coupling ratio of the dark matter particle. As emphasized for example in [12], the interpretation of the experimental results may substantially change within this more general isospin-violating scenario.

Recently, the DEAP collaboration reported the results of a dark matter search, based on 231-live days of data taken during the first year of operation, with the DEAP-3600 experiment [13]. DEAP-3600 is a direct-detection dark matter experiment that uses $3279 \mathrm{~kg}$ of liquid argon as target and is located $2 \mathrm{~km}$ underground at SNOLAB. At first sight, these results seem to provide no new constraints on the dark matter interactions, for the limit on the dark matter-nucleon spin independent cross section lies well inside the region already excluded by three different xenon experiments: LUX [5], PandaX-II [6], and XENON1T [4]. In this paper, we point out that this conclusion is not entirely correct. We reexamine these constraints for isosping-violating dark matter and find that there are regions in the parameter space where the DEAP-3600 limit is up to a factor of two stronger than that from XENON1T. These regions, which we delimit and characterize, correspond to the so-called Xenonphobic dark matter scenario [14], for which the neutron-to-proton coupling ratio is close to -0.7 . Our results seem to signal the end of the xenon-dominated epoch and the beginning of a new era in which the complementarity among different direct detection targets will be essential in the determination of the fundamental properties of the dark matter particle.

The rest of the manuscript is organized as follows. In the next section, the main features of the isospin-violating scenario are briefly reviewed. Section 3 presents our main results. In it we compare the limits from DEAP-3600 and XENON1T for isospin-violating dark matter. Finally, our conclusions are drawn in section 4 .

\section{Theoretical Framework}

For completeness, the isospin-violating scenario for dark matter [7-11] is briefly reviewed in this section. In particular, we analyze how the interpretation of the experimental results changes within this more general framework. We refer the reader to [12] for a more general discussion and additional references.

The dark matter-nucleus spin-independent cross section, $\sigma_{A_{i}}$, is generally given by

$$
\sigma_{A_{i}}=\frac{4 \mu_{A_{i}}^{2}}{\pi}\left[f_{p} Z+f_{n}\left(A_{i}-Z\right)\right]^{2},
$$

where $\mu_{A_{i}}=M_{D M} M_{A_{i}} /\left(M_{D M}+M_{A_{i}}\right)$ is the dark matter-nucleus reduced 
mass, $A_{i}$ is the number of nucleons, and $Z$ is the nucleus charge. $f_{p}$ and $f_{n}$ denote the dark matter coupling to the proton and the neutron respectively, and are determined by the underlying particle physics model that accounts for the dark matter. Notice, in particular, that a priori there is no reason to expect $f_{p}=f_{n}$ (It is known, though, that the assumptions of Majorana dark matter and minimal flavor violation lead to $\left.f_{p} \approx f_{n}[15]\right)$. That is, in general the dark matter particle will couple differently to protons and neutrons. In fact, several models for which $f_{n} / f_{p} \neq 1$ have been studied in the literature [16-23]. Theoretically motivated scenarios with isospin violation include models where the dark matter interactions are mediated by a dark photon, by the $Z^{0}$ boson, or by squarks [15]. Thus, it is just a historical accident that the peculiar case $f_{n} / f_{p}=1$ has become the default dark matter scenario while the more general case $f_{n} / f_{p} \neq 1$ is considered special and is referred to as isospin-violating dark matter.

Another relevant quantity is the dark matter-proton spin-independent cross section, $\sigma_{p}$, which is given by

$$
\sigma_{p}=\frac{4 \mu_{p}^{2}}{\pi} f_{p}^{2} .
$$

Direct detection experiments, however, typically report their exclusion limits in terms of the so-called dark matter-nucleon cross section, $\sigma_{N}^{Z}$, which can be written as

$$
\sigma_{N}^{Z}=\sigma_{p} \frac{\sum_{i} \eta_{i} \mu_{A_{i}}^{2}\left[Z+\left(A_{i}-Z\right) f_{n} / f_{p}\right]^{2}}{\sum_{i} \eta_{i} \mu_{A_{i}}^{2} A_{i}^{2}} .
$$

For $f_{p}=f_{n}$ (isospin-conservation), as assumed in most experimental reports, $\sigma_{N}^{Z}$ and $\sigma_{p}$ coincide, but in general this is not the case. In particular, if $f_{p}$ and $f_{n}$ have opposite signs $\left(f_{n} / f_{p}<0\right)$, one can have destructive interference between the proton and the neutron contributions to the cross section, so that $\sigma_{N}^{Z} \ll \sigma_{p}$. In general, it is $\sigma_{p}$, rather than $\sigma_{N}^{Z}$, that is physically meaningful and that should be used to present and compare different experimental results. It is useful, therefore, to define the ratio between these two quantities,

$$
F_{Z} \equiv \frac{\sigma_{p}}{\sigma_{N}^{Z}} .
$$

$F_{Z}$, which depends on the target nucleus and on $f_{n} / f_{p}$, gives the factor by which the sensitivity of a direct detection experiment is suppressed for isospinviolating dark matter. In other words, if $\tilde{\sigma}$ is the limit on the spin-independent dark matter-nucleon cross section reported by an experiment (at a given dark matter mass), then $F_{Z} \tilde{\sigma}$ is the limit on the dark matter-proton cross section that actually applies to the isospin-violating scenario.

Figure 1 shows $F_{Z}$ for xenon (dashed line) and argon (solid line), and for values of $f_{n} / f_{p}$ between -1 and 0 . Because argon consists mostly of a single isotope, there exists a value of $f_{n} / f_{p}$ for which there is an exact cancellation between the neutron and proton contributions to the cross section, so that the dark matter does not interact with an argon nucleus. At such point, $f_{n} / f_{p} \approx$ 


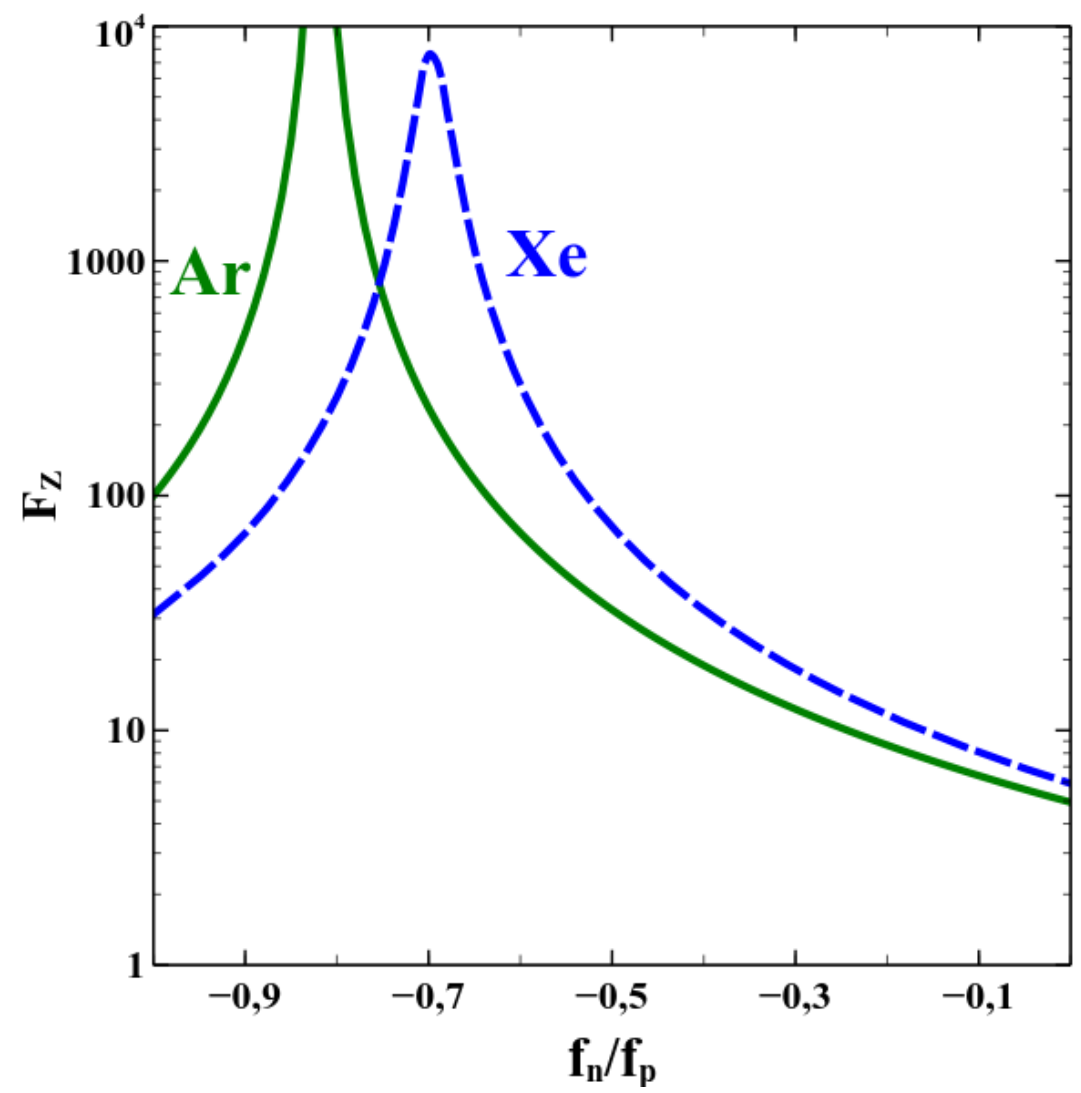

Figure 1: The suppression factor, $F_{Z}$, as a function of $f_{n} / f_{p}$ for xenon (blue) and argon (green) targets. $F_{Z}$ gives the sensitivity loss on the dark matter-proton spin-independent cross section for the case of isospin-violating dark matter.

-0.82 , argon experiments completely lose their sensitivity $\left(F_{Z} \rightarrow \infty\right)$, as shown in the figure.

On the other hand, since xenon is composed of several isotopes, an exact cancellation is not possible and $F_{Z}$ has a maximum value -the relevance of the distribution of isotopes present in each detector was first emphasized in [11]. This maximum value of $F_{Z}$ is achieved for $f_{n} / f_{p} \approx-0.7$ and amounts to about $10^{4}$. Xenonphobic dark matter is defined as a dark matter particle featuring a value of $f_{n} / f_{p}$ in the vicinity of -0.7 [14] ${ }^{1}$. Notice that, although highly suppressed, the coupling between a xenon nucleus and a Xenonphobic dark matter particle is not zero.

According to figure 1 , for Xenonphobic dark matter $\left(f_{n} / f_{p} \approx-0.7\right)$, the limits from xenon experiments (XENON1T, PandaX-II, LUX) are actually weaker

\footnotetext{
${ }^{1}$ It was called Xenophobic in that work; we have used instead the more precise Xenonphobic.
} 


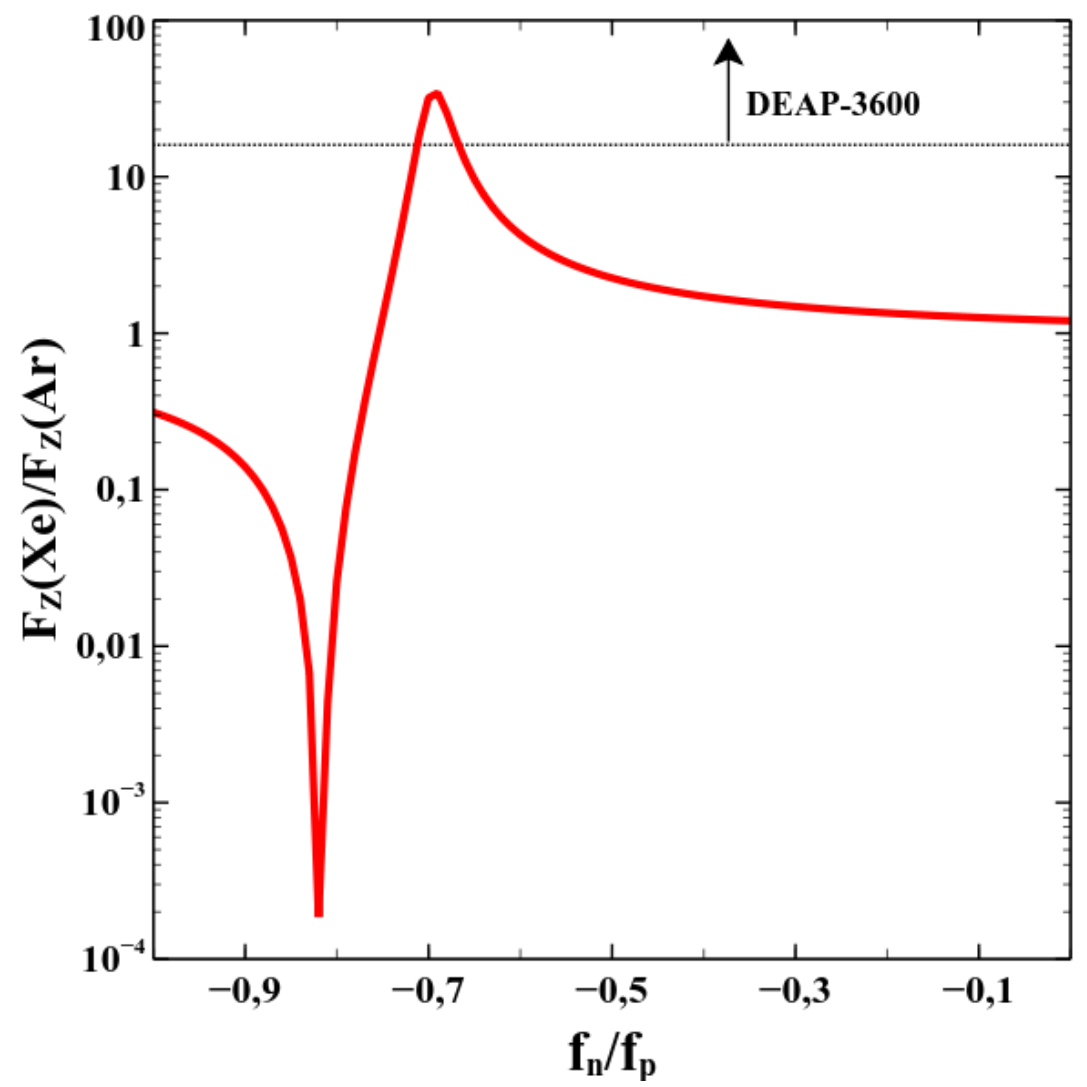

Figure 2: The values of $f_{n} / f_{p}$ where DEAP-3600 outperforms XENON1T for high dark matter masses. Above the dotted black line, DEAP-3600 currently provides the most stringent limit on the dark matter spin-independent cross section.

by almost four orders of magnitude whereas those from argon experiments by about two orders of magnitude. Since the gap between the 2018 XENON1T limit and the recent limit from DEAP-3600 can be less than two orders of magnitude, the latter may set the most stringent constraints on Xenonphobic dark matter. This fact is illustrated in figure 2, which shows the ratio between the $F_{Z}$ factors for xenon and argon (solid line), and compares it against the ratio of the limits from XENON1T and DEAP-3600 at high dark matter masses (dotted horizontal line). Above the dotted line, for $f_{n} / f_{p}$ in the Xenonphobic region, DEAP-3600 is expected to set the most stringent limits for large dark matter masses. In the next section, the region of parameter space where DEAP-3600 outperforms XENON1T is determined. 


\section{Results}

To begin with, let us illustrate the latest results from DEAP-3600 and how they compare against other experiments under the standard assumption, $f_{n} / f_{p}=1$ -see figure 3. This figure is quite similar to that shown by the DEAP collaboration in their recent publication [13]. It displays the current limits on the dark matter-nucleon spin-independent cross section from different experiments: DS-50 (dotted line), DEAP-3600 (solid line), LUX (short-dashed line), PandaX-II (dash-dotted line) and XENON1T (dashed line). The upper two lines correspond to argon experiments whereas the lower three lines to xenon experiments. From this figure it seems that the new results from DEAP-3600 are hardly relevant; they are just excluding a region that had already been excluded by three different experiments. In the following, we will challenge this interpretation.

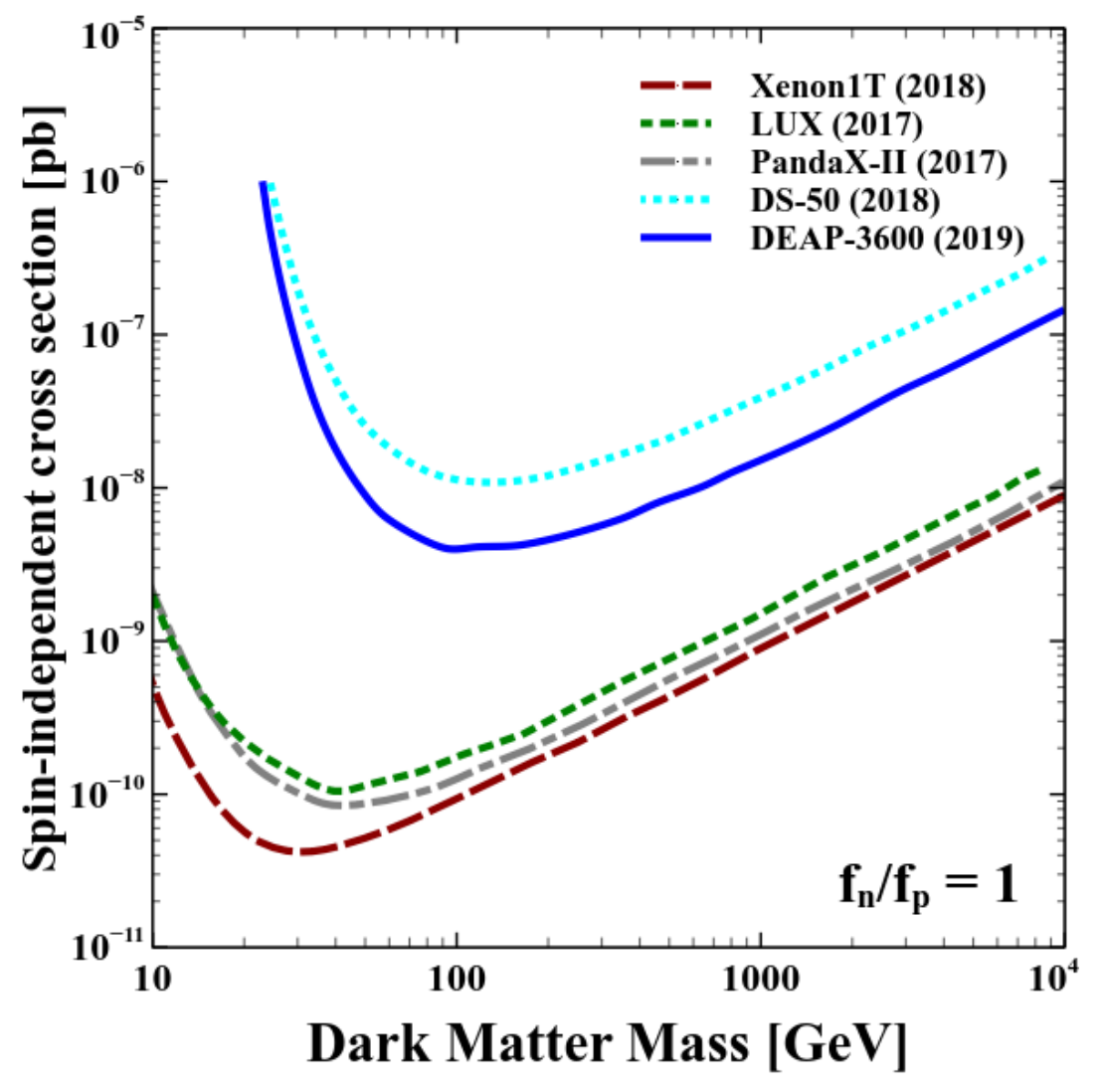

Figure 3: Current limits on the dark matter-proton spin-independent direct detection cross section for the case $f_{n} / f_{p}=1$ (isospin-conserving dark matter). 


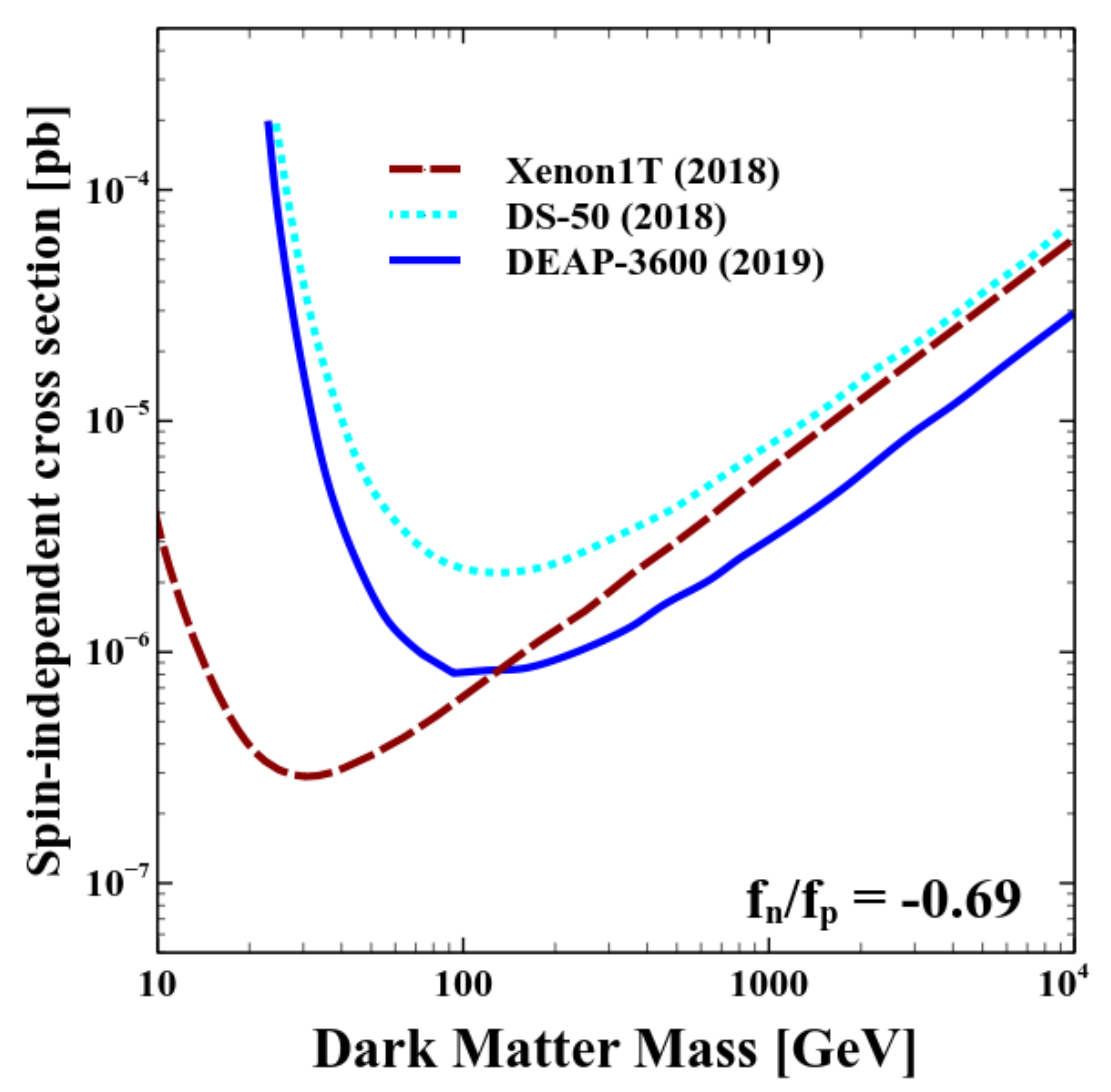

Figure 4: Current limits on the dark matter-proton spin-independent direct detection cross section for the case $f_{n} / f_{p}=-0.69$ (isospin-violating dark matter).

The crucial point is that figure 3 is valid only for $f_{n} / f_{p}=1$, an assumption without any theoretical or experimental support. And as explained in the previous section, the interpretation of these experimental results may drastically change when we consider the more general scenario of isospin-violating dark matter. In fact, we already know that it is for Xenonphobic dark matter $\left(f_{n} / f_{p} \approx-0.7\right)$ that we expect the most significant modifications.

Figure 4 compares the experimental limits from XENON1T, DS-50, and DEAP-3600 for $f_{n} / f_{p}=-0.69$. For clarity we dropped from this figure the limits from LUX and PandaX-II, which are always close to and weaker than the XENON1T limit. Notice, first of all, that the scale on the $y$ axis is different from the previous figure, as all limits become weaker -see figure 1. Remarkably, we find that, in this case, the recent limit from DEAP-3600 is actually stronger than that from XENON1T (or any other xenon experiment) in the region $M_{D M} \gtrsim 130$ $\mathrm{GeV}$. At high masses, the difference between these two limits amounts to about 


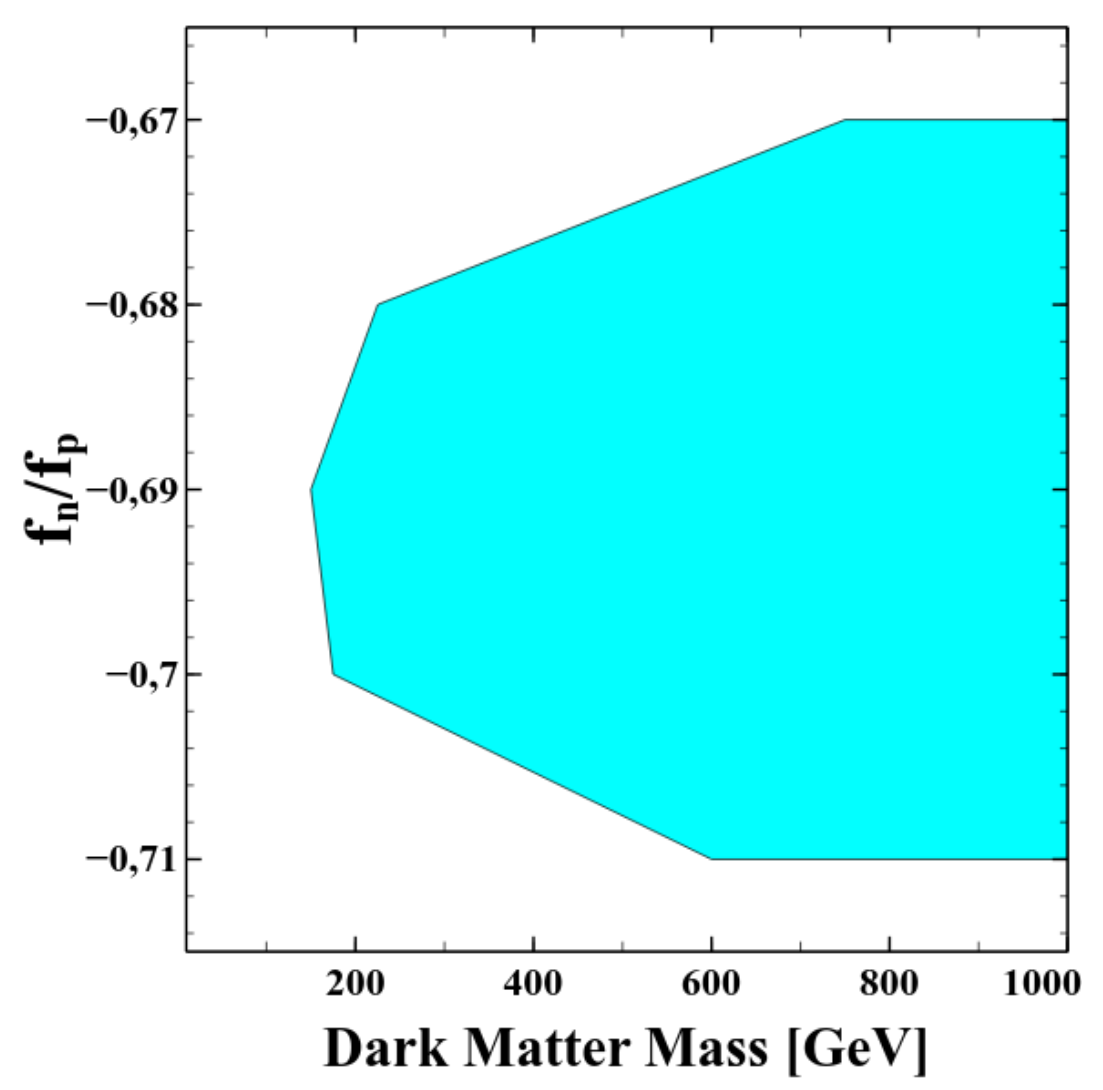

Figure 5: The region of parameter space where DEAP-3600 currently sets the most stringent limits on the dark matter-proton spin-independent cross section.

a factor of two. That is, the recent results from DEAP-3600 are actually probing new regions of the parameter space for Xenonphobic dark matter.

This figure not only demonstrates the main thesis of this paper but it also emphasizes the need to find a better way to present and compare the limits (and future signals) from direct detection experiments using different targets. At the very least, a caveat should be included indicating that the result is valid only for the special case $f_{n} / f_{p}=1$.

Figure 5 displays the region of parameter space where DEAP-3600 currently outperforms XENON1T. It includes values of $f_{n} / f_{p}$ between -0.67 and -0.71 , and dark matter masses larger than about $150 \mathrm{GeV}$. Given that both experiments are expected to release new limits in the near future, it will be interesting to see how this region gets updated.

In figure 4 we also displayed the recent limit from DS-50 (dotted line), another argon experiment, because we wanted to make a point about the qual- 
itative change that has occurred with the first-year limit from DEAP-3600. Notice that the region excluded by DS-50 lies entirely within the XENON1T exclusion region. Thus, DS-50 is not probing new regions of the parameter space. In other words, until the recent limit from DEAP-3600, xenon experiments were so dominant that they were imposing the most stringent constraints even on Xenonphobic dark matter -see also [12]. It is only now, with the release of the first-year of data from DEAP-3600, that this situation has changed. This new development -the fact that argon experiments have already become competitive- is essential, for it may allow to test, once dark matter signals are observed, if the dark matter interactions are really isospin-conserving $\left(f_{p}=f_{n}\right)$ or not -see e.g. [15] for a recent discussion. The observation of dark matter direct detection signals from different targets is also required to test whether the dark matter particle is its own antiparticle, as proposed in [24,25]. Our results seem to indicate that we have just entered into a new era in which the complementarity among different direct detection targets will play a crucial role in the determination of the fundamental properties of the dark matter particle.

\section{Conclusions}

We demonstrated that the first-year results from DEAP-3600, a single-phase liquid argon direct-detection dark matter experiment, are more relevant than they appear at first sight, for they set new limits on Xenonphobic dark matter $\left(f_{n} / f_{p} \approx-0.7\right)$. In fact, the DEAP-3600 limit may exceed by up to a factor of two the current limits from xenon experiments (see figure 4). In this work, the range of parameter space for which DEAP-3600 sets the most stringent limits on the dark matter-proton spin-independent cross section was delimited (see figure 5). Finally, we also pointed out that these new limits seem to signal the end of the xenon-dominated epoch and the beginning of a new era in which the complementarity among different direct detection targets will play a crucial role in the determination of the dark matter nature.

\section{References}

[1] Jonathan L. Feng. Dark Matter Candidates from Particle Physics and Methods of Detection. Ann. Rev. Astron. Astrophys., 48:495-545, 2010, 1003.0904 .

[2] Giorgio Arcadi, Maíra Dutra, Pradipta Ghosh, Manfred Lindner, Yann Mambrini, Mathias Pierre, Stefano Profumo, and Farinaldo S. Queiroz. The waning of the WIMP? A review of models, searches, and constraints. Eur. Phys. J., C78(3):203, 2018, 1703.07364.

[3] Teresa Marrodán Undagoitia and Ludwig Rauch. Dark matter directdetection experiments. J. Phys., G43(1):013001, 2016, 1509.08767. 
[4] E. Aprile et al. Dark Matter Search Results from a One Ton-Year Exposure of XENON1T. Phys. Rev. Lett., 121(11):111302, 2018, 1805.12562.

[5] D. S. Akerib et al. Results from a search for dark matter in the complete LUX exposure. Phys. Rev. Lett., 118(2):021303, 2017, 1608.07648.

[6] Xiangyi Cui et al. Dark Matter Results From 54-Ton-Day Exposure of PandaX-II Experiment. Phys. Rev. Lett., 119(18):181302, 2017, 1708.06917.

[7] Andriy Kurylov and Marc Kamionkowski. Generalized analysis of weakly interacting massive particle searches. Phys. Rev., D69:063503, 2004, hep$\mathrm{ph} / 0307185$.

[8] F. Giuliani. Are direct search experiments sensitive to all spin-independent WIMP candidates? Phys. Rev. Lett., 95:101301, 2005, hep-ph/0504157.

[9] Spencer Chang, Jia Liu, Aaron Pierce, Neal Weiner, and Itay Yavin. CoGeNT Interpretations. JCAP, 1008:018, 2010, 1004.0697.

[10] Zhaofeng Kang, Tianjun Li, Tao Liu, Chunli Tong, and Jin Min Yang. Light Dark Matter from the $U(1)_{X}$ Sector in the NMSSM with Gauge Mediation. JCAP, 1101:028, 2011, 1008.5243.

[11] Jonathan L. Feng, Jason Kumar, Danny Marfatia, and David Sanford. Isospin-Violating Dark Matter. Phys. Lett., B703:124-127, 2011, 1102.4331.

[12] Carlos E. Yaguna. Isospin-violating dark matter in the light of recent data. Phys. Rev., D95(5):055015, 2017, 1610.08683.

[13] R. Ajaj et al. Search for dark matter with a 231-day exposure of liquid argon using DEAP-3600 at SNOLAB. 2019, 1902.04048.

[14] Jonathan L. Feng, Jason Kumar, and David Sanford. Xenophobic Dark Matter. Phys. Rev., D88(1):015021, 2013, 1306.2315.

[15] Chris Kelso, Jason Kumar, Danny Marfatia, and Pearl Sandick. Directly detecting Isospin-Violating Dark Matter. Phys. Rev., D97(5):056004, 2018, 1711.01644 .

[16] Koichi Hamaguchi, Seng Pei Liew, Takeo Moroi, and Yasuhiro Yamamoto. Isospin-Violating Dark Matter with Colored Mediators. JHEP, 05:086, 2014, 1403.0324 .

[17] Aleksandra Drozd, Bohdan Grzadkowski, John F. Gunion, and Yun Jiang. Isospin-violating dark-matter-nucleon scattering via two-Higgs-doubletmodel portals. 2015, 1510.07053 .

[18] Mads T. Frandsen, Felix Kahlhoefer, Subir Sarkar, and Kai SchmidtHoberg. Direct detection of dark matter in models with a light Z'. JHEP, 09:128, 2011, 1107.2118. 
[19] Geneviève Bélanger, Andreas Goudelis, Jong-Chul Park, and Alexander Pukhov. Isospin-violating dark matter from a double portal. JCAP, 1402:020, 2014, 1311.0022.

[20] Víctor Martín Lozano, Miguel Peiró, and Pablo Soler. Isospin violating dark matter in Stückelberg portal scenarios. JHEP, 04:175, 2015, 1503.01780.

[21] Xin Gao, Zhaofeng Kang, and Tianjun Li. Origins of the Isospin Violation of Dark Matter Interactions. JCAP, 1301:021, 2013, 1107.3529.

[22] Jonathan L. Feng, Jason Kumar, Danny Marfatia, and David Sanford. Isospin-Violating Dark Matter Benchmarks for Snowmass 2013. In Proceedings, Community Summer Study 2013: Snowmass on the Mississippi (CSS2013): Minneapolis, MN, USA, July 29-August 6, 2013, 2013, 1307.1758.

[23] Nobuchika Okada and Osamu Seto. Isospin violating dark matter being asymmetric. Phys. Rev., D88:063506, 2013, 1304.6791.

[24] Farinaldo S. Queiroz, Werner Rodejohann, and Carlos E. Yaguna. Is the dark matter particle its own antiparticle? Phys. Rev., D95(9):095010, 2017, 1610.06581 .

[25] Bradley J. Kavanagh, Farinaldo S. Queiroz, Werner Rodejohann, and Carlos E. Yaguna. Prospects for determining the particle/antiparticle nature of WIMP dark matter with direct detection experiments. JHEP, 10:059, 2017, 1706.07819. 